\title{
Traffic-Balanced Routing Algorithm for Irregular Mesh-Based On-Chip Networks
}

\author{
Shu-Yen Lin, Chun-Hsiang Huang, Chih-Hao Chao, \\ Keng-Hsien Huang, and An-Yeu (Andy) Wu, Member, IEEE
}

\begin{abstract}
On-chip networks (OCNs) have been proposed to solve the increasing scale and complexity of the designs in nanoscale multicore VLSI designs. The concept of irregular meshes is an important issue because IPs of different sizes may be supported by various vendors. In order to solve routing problems in irregular meshes, modified routing algorithms to detour oversized IPs (OIPS) are needed. However, directly applying fault-tolerant routing algorithms may cause two serious problems: 1) heavy traffic loads around OIPs and 2) unbalanced traffic loads in irregular meshes. In this paper, we propose an OIP Avoidance Prerouting (OAPR) algorithm to solve the aforementioned problems. The proposed OAPR can make traffic loads evenly spread on the networks and shorten the average paths of packets. Therefore, the networks using the OAPR have lower latency and higher throughput than those using faulttolerant routing algorithms. In our experiments, four different cases are simulated to demonstrate that the proposed OAPR improves 13.3 percent to 100 percent sustainable throughputs than two previous fault-tolerant routing algorithms. Moreover, the hardware overhead of the OAPR is less than 1 percent compared to the cost of a whole router. Hence, the proposed OAPR algorithm has good performance and is practical for irregular mesh-based OCNs.
\end{abstract}

Index Terms-Fault tolerance, network topology, routing protocols.

\section{INTRODUCTION}

S YSTEM-ON-CHIP (SoC) designs provide the integrated solution to the complex VLSI designs. However, in deep submicron (DSM) technology, the existing on-chip interconnection faces many challenges due to the increasing scale and complexity of the designs. Recently, on-chip networks $(\mathrm{OCNs})$ have been proposed as a solution for the problems [1], [2], [3], [4]. Kumar et al. presented a design methodology specifically for a 2D-mesh OCN [5]. Numerous researches are based on 2D meshes because of their regularity. In $2 \mathrm{D}$ meshes, each router is connected to a single IP such as a CPU, a DSP core, or an embedded memory. However, the assumption that each IP has the same size is not practical. The sizes of hard IPs from various vendors may be different (such as an ARM processor, a CPU, a MPEG4 decoder, etc.). In order to apply the hard IPs to 2D meshes, the concept of irregular meshes for placing Oversized IPs (OIPs) has been proposed in [6], [7].

Fig. 1a shows a conventional $6 \times 62 \mathrm{D}$ mesh. Each router in the $2 \mathrm{D}$ mesh is connected to a normal-sized IP. Fig. $1 \mathrm{~b}$ shows a $6 \times 6$ irregular mesh with 1 OIP and 31 normalsized IPs. The placement of the OIP eliminates some routing paths of the regular $2 \mathrm{D}$ mesh; hence, existing routing algorithms on 2D meshes may fail. Some direct approaches, such as using extra physical links in the

- The authors are with the Department of Electrical Engineering, National Taiwan University, R323, EE-2, No. 1, Sec. 4, Roosevelt Road, Taipei, 106, Taiwan (ROC).

E-mail: \{linyan, ajiow, chihhao, time\}@access.ee.ntu.edu.tw, andywu@cc.ee.ntu.edu.tw.

Manuscript received 2 July 2007; revised 14 Dec. 2007; accepted 13 Mar. 2008; published online 4 Apr. 2008.

Recommended for acceptance by R. Marlescu.

For information on obtaining reprints of this article, please send e-mail to: tc@computer.org, and reference IEEECS Log Number TCSI-2007-07-0297. Digital Object Identifier no. 10.1109/TC.2008.60. surroundings of OIPs or scaling the physical channels around OIPs, may solve the OIP problem. However, these extra links and channels around the OIPs may cause more serious crosstalk, noise, and delay problems in deep submicron VLSI technologies, as pointed out in [1], [19], [20]. Therefore, modified routing algorithms of the $2 \mathrm{D}$ mesh are usually employed to solve the OIP problem in the literature. In [7] and [11], two fault-tolerant routing algorithms, the Extended $X-Y$ routing algorithm (E-XY) [10] and Chen and Chiu's algorithm (Chen and Chiu's) [8] (the deadlock problem in [8] was corrected in [17]), are directly applied on irregular-mesh problems. Fault-tolerant routing algorithms are workable because of the similarity between faulty networks and on-chip irregular meshes. However, fault-tolerant routing algorithms are not suitable for irregular meshes. Directly applying fault-tolerant routing algorithms causes heavy traffic loads around the OIP and unbalanced traffic in the networks. The abilities of detouring faulty blocks are the major concern for fault-tolerant routing algorithms, while performance issues are considered for the routings in irregular meshes. In this paper, we propose an OIP Avoidance Prerouting (OAPR) algorithm based on the odd-even turn model [13] for routings in irregular meshes without extra virtual channels. Fig. 2 shows the concept of the OAPR from the experimental results in Figs. 20b, 20c, and 20d. If we apply the E-XY [10] and Chen and Chiu's [8], traffic loads around the OIPs are huge and unbalanced, as shown in Figs. 2a and $2 \mathrm{~b}$. However, our proposed OAPR algorithm results in lower and more balanced traffic loads around the OIPs (Fig. 2c) because it can avoid the routing paths around the OIPs and takes all usable turns in the odd-even turn model. Therefore, the networks using the OAPR have better performance than those using Chen and Chiu's [8] and the E-XY [10].

In [9], the concepts of the fault ring (f-ring) and the fault chain (f-chain) are proposed according to the positions of 


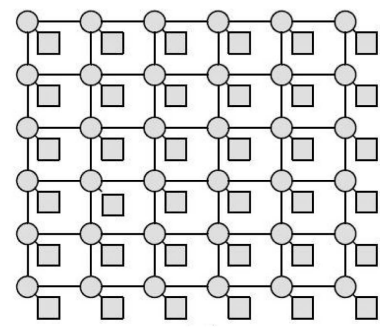

(a)

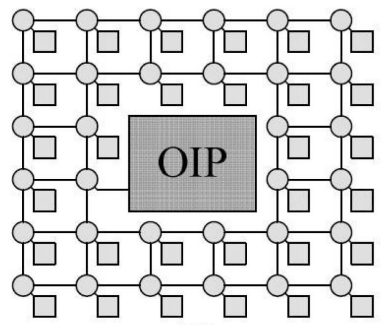

(b)
Router Normal-sized IP

Oversized IP

Fig. 1. (a) A conventional $6 \times 62 \mathrm{D}$ mesh and (b) a $6 \times 6$ irregular mesh with 1 OIP and 31 normal-sized IPs.

faulty blocks. An f-ring is a faulty region enclosed by a set of nonfaulty routers. An f-chain is a faulty region located at the boundaries of mesh networks. Fig. 3a shows an example of one f-ring and one f-chain. The disabled routers in faulty blocks cannot be used in routing process. Besides, f-chains can be classified into eight different types according to the boundaries of a mesh network, called NW-chains, NE-chains, SW-chains, SE-chains, N-chains, S-chains, E-chains, and $W$-chains. Fig. $3 b$ shows an example of one f-ring and eight different types of $\mathrm{f}$-chains in a $10 \times 10$ mesh. According to the classification in Fig. 3b, Chen and Chiu's [8] can support the placements of f-rings and f-chains, but the E-XY [10] only supports f-rings. However, the proposed OAPR can support the placements of f-rings and $\mathrm{f}$-chains to reduce the restrictions of OIP placements (the placements of oversized IPs).

In summary, the OAPR contains four routing modes:

1. Default Routing,

2. Single OIP,

3. Multiple OIPs, and

4. f-chain.

Routing modes 1, 2, and 3 are routing rules for f-rings. Routing mode 4 shows extra rules for f-chains. Each routing mode is based on the odd-even turn model to prevent deadlock. The routing is more generalized if more routing modes are supported. It means that the routing can support more different kinds of OIP placed.

In our simulation, we introduce two methods, 1) static analysis and 2) dynamic simulation, to evaluate the performance of the OAPR. Static analysis uses statistical information to evaluate network performance quickly. In dynamic simulation, we simulate not only the random traffic pattern but also the exponential and the Rent's rule

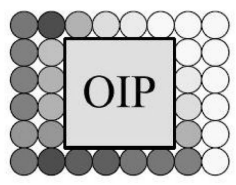

(a)

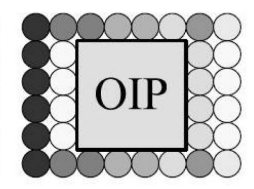

(b)

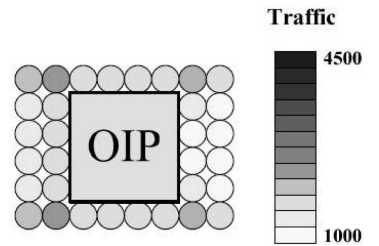

(c)
Fig. 2. Traffic loads around the OIPs by using (a) Chen and Chiu's algorithm [8] (unbalanced), (b) the E-XY [10] (unbalanced), and (c) the OAPR (balanced).

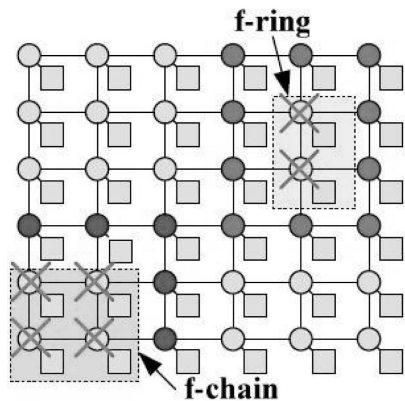

(a)

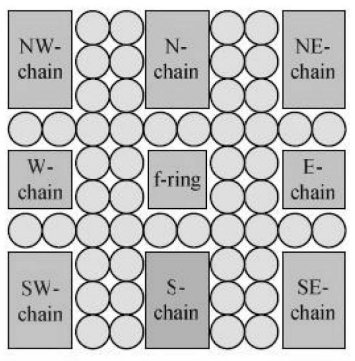

(b)
Fig. 3. Two examples of f-rings and f-chains. (a) One f-ring and one $\mathrm{f}$-chain in a $6 \times 6$ mesh and (b) one f-ring and eight different types of $\mathrm{f}$-chains in a $10 \times 10$ mesh.

[16] traffic patterns recently mentioned in NOCS 2007. Four different cases are simulated to show that the networks using the OAPR improve 13.3 percent to 100 percent throughput than those using the E-XY [10] and Chen and Chiu's [8].

Our proposed OAPR can solve three routing problems in irregular meshes:

- heavy traffic loads around boundaries of OIPs,

- unbalanced traffic loads in irregular meshes, and

- OIPs can be placed at f-rings and f-chains, which are allowed in [8] but restricted in [10].

The rest of this paper is organized as follows: In Section 2, we briefly review the basic topology-2D mesh, the oddeven turn model, and two previous fault-tolerant routing algorithms. In Section 3, the proposed OAPR algorithm for irregular meshes is introduced. In Section 4, we evaluate the performance of the E-XY [10], Chen and Chiu's [8], and the OAPR by two methods: static analysis and dynamic simulations. In Section 5, the steps to implement our algorithm are described. Finally, the conclusion is shown in Section 6.

\section{Review of Previous Works}

In this section, we first review the mesh topology and Chiu's odd-even turn model [13], which is an extension of Glass and Ni's turn model [12]. After that, we discuss Wu's Extended XY routing [10] and Chen and Chiu's faulttolerant routing [8]. Both of these fault-tolerant routing algorithms belong to deterministic routings and avoid deadlock without extra virtual channels.

\subsection{D Meshes}

An $n \times n$ two-dimensional mesh (2D mesh) contains $n^{2}$ routers. Each router has an address $(x, y)$, where $x$ and $y \in\{0,1, \ldots, n-1\}$. We define the coordinate $x(y)$ increasing along the east (north) direction. Therefore, the router located in the southwest corner of the 2D mesh has an address $(0,0)$ and the router placed in the northeast corner has an address $(n-1, n-1)$. Each router in 2D mesh contains five ports: four ports connected to neighbor routers (north, east, south, and west) except for the routers located in the boundaries of 2D meshes and one port linked to a local IP. Besides, two routers, $u:\left(u_{x}, u_{y}\right)$ 


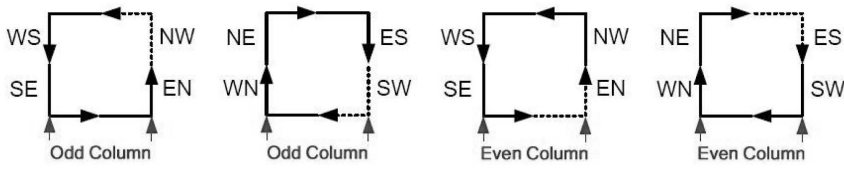

Fig. 4. The odd-even turn model.

and $v:\left(v_{x}, v_{y}\right)\left(u_{x}, u_{y}, v_{x}\right.$, and $v_{y} \in\{0,1, \ldots, n-1\}$ in an $n \times n$ mesh), are connected if the addresses differ in only coordinate $x$ or $y$ and the difference is equal to 1 . In other words, $u$ and $v$ conform to either $\left\{\left|u_{x}-v_{x}\right|=1, u_{y}=v_{y}\right\}$ or $\left\{\left|u_{y}-v_{y}\right|=1, u_{x}=v_{x}\right\}$ if they are neighbors.

\subsection{The Odd-Even Turn Model [13]}

In [13], Chiu extended the idea from Glass and Ni's turn model [12] and proposed the odd-even turn model. The odd-even turn model avoids deadlock by prohibiting two turns for odd and even columns and performs fairer routing adaptiveness. Fig. 4 shows the restricted turns of the odd-even turn model. In odd columns, south-to-west (SW) turns and north-to-west (NW) turns are avoided. In even columns, east-to-south (ES) turns and east-to-north (EN) turns are restricted. The odd-even turn model prevents the formation of the rightmost column of a cycle. Besides, 180-degree turns are prohibited in the odd-even turn model.

\subsection{The Extended X-Y Routing [10]}

In [10], $\mathrm{Wu}$ proposed $E-X Y$ routing algorithm based on the dimension-order routing and the odd-even turn model [13]. The E-XY can avoid deadlock without virtual channels. The E-XY consists of two modes: 1) normal and 2) abnormal mode. In normal mode, the E-XY is similar to the dimension-order routing and only turns at even columns if no faulty block obstructs the routing paths, as shown in Fig. 5. Otherwise, the abnormal mode shown in Fig. 6 is selected. The symbol $E(O)$ means that the E-XY turns at even (odd) columns. The FBs stand for faulty blocks in Figs. 5, 6, and 7. However, the drawbacks are shown as follows:

- Traffic loads on even columns are more serious than odd columns: The E-XY only turns at even columns in normal mode.

- Traffic loads on the boundaries of faulty blocks are heavy and unbalanced: The routing paths follow the boundaries of faulty blocks in abnormal mode. Moreover, traffic loads on west boundaries of faulty

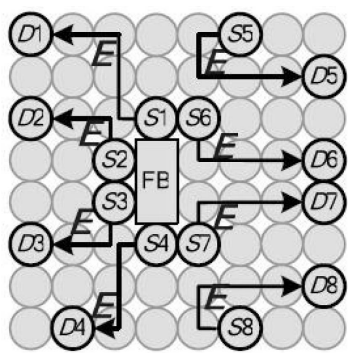

Fig. 5. Eight possible cases of the E-XY in normal mode.

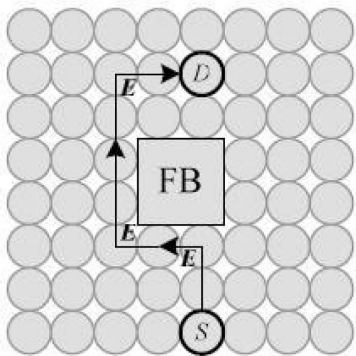

(a)

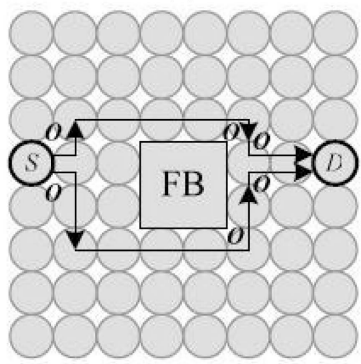

(c)

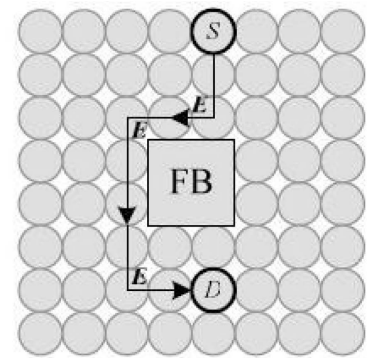

(b)

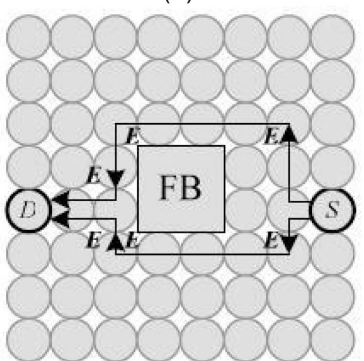

(d)
Fig. 6. Four cases of the E-XY in abnormal mode: (a) south-to-north, (b) north-to-south, (c) west-to-east, and (d) east-to-west direction.

blocks are heavier than east boundaries, as seen in the experimental results in Figs. 20b and 23b.

- The E-XY cannot solve faulty blocks located at the boundaries of 2D meshes: In the E-XY, each fault block must be surrounded by a boundary ring. The boundary ring consists of six lines: two lines at the east side, two lines at the west side, one line at the north side, and one line at the south side.

\subsection{Chen and Chiu's Algorithm [8]}

Chen and Chiu proposed their fault-tolerant routing algorithm (Chen and Chiu's) in [8]. Chen and Chiu's prohibited some turns to avoid the formation of the rightmost column segment of a circular waiting path. Hence, the algorithm can solve deadlock without using virtual channels. In addition, they employed the concepts of the $f$-ring and the $f$-chain according to the positions of faulty blocks [9]. The routings around the f-chains and f-rings are available in Chen and Chiu's.

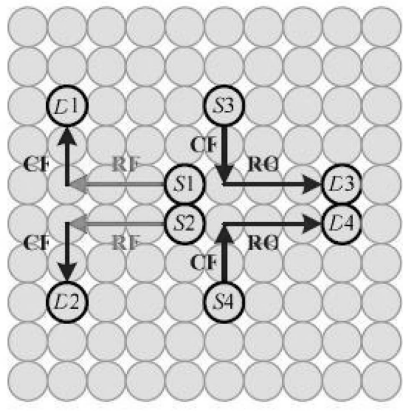

(a)

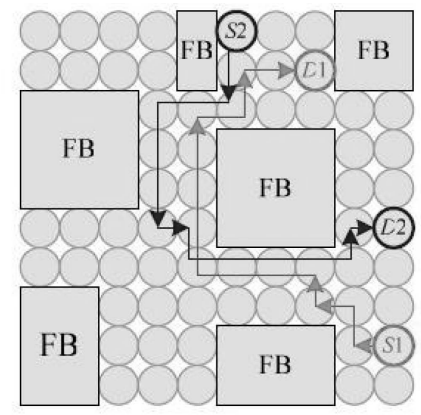

(b)
Fig. 7. Examples of Chen and Chiu's routing algorithm: (a) the routing paths (RF, CF, and RO) in Normal-Route and (b) two examples of RingRoute and Chain-Route. 


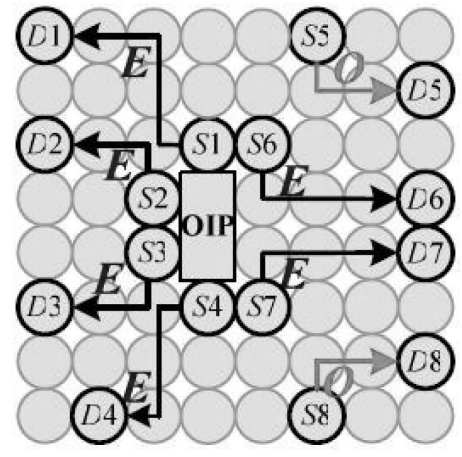

Fig. 8. Eight possible cases of Default routing.

Chen and Chiu's contains three different modes: 1) Normal-Route, 2) Ring-Route, and 3) Chain-Route. In Normal-Route, row-first $(R F)$, column-first $(C F)$, and rowonly $(R O)$ routing paths are used. Fig. 7a shows some possible routing paths for $\mathrm{RF}, \mathrm{CF}$, and $\mathrm{RO}$. If faulty blocks are encountered, Ring-Route or Chain-Route is executed to misroute the f-rings and the f-chains. Fig. $7 \mathrm{~b}$ shows two examples ( $S 1$ to $D 1$ and $S 2$ to $D 2$ ) to misroute the f-rings and f-chains. The detailed descriptions are discussed in [8].

However, Chen and Chiu's still has a drawback: Traffic loads around faulty blocks are heavy and unbalanced, as seen in the experimental results in Figs. 20c, 23c, and 25b. The routing paths are asymmetric and along the boundaries of faulty blocks.

Therefore, directly applying the E-XY and Chen and Chiu's algorithms in irregular meshes would cause serious contention. In Section 3, we proposed an OAPR algorithm to solve the problems mentioned in this section.

\section{Proposed OAPR Routing Algorithm for IRREGULAR MESHES}

The proposed OIP-Avoidance Prerouting (OAPR) algorithm is introduced in this section. The OAPR has two major features, described as follows:

1. Avoid routing paths along boundaries of OIPs. In the environment of faulty meshes, we can only know the information of faulty blocks in real time. However, the locations of OIPs are known in advance. Therefore, the OAPR can avoid routing paths along boundaries of OIPs and reduce the traffic loads around OIPs. With these features, the OAPR can achieve more balanced traffic loads in irregular meshes.

2. Support f-rings and f-chains for placements of OIPs. The OAPR solves the drawbacks of the E-XY and uses the odd-even turn model to avoid deadlock systematically. However, the E-XY cannot support OIPs placed at boundaries of irregular meshes. In order to solve this problem, the OAPR applies the concepts of f-rings and f-chains [9]. With this feature, the OAPR can work correctly if OIPs are placed at the boundaries of irregular meshes.

The OAPR contains four routing modes:

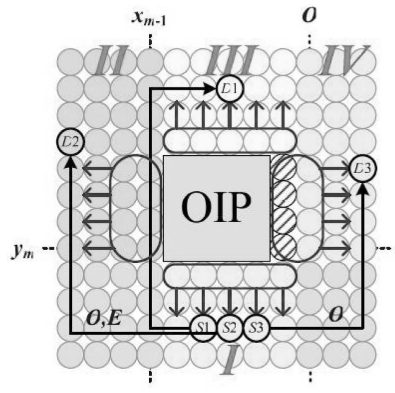

(a)

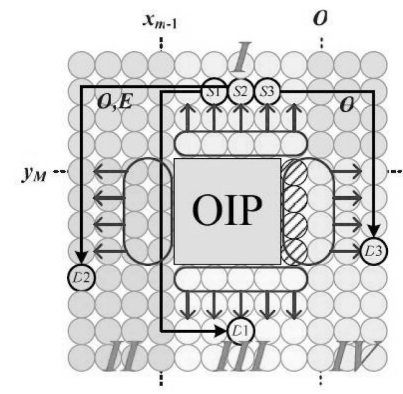

(b)
Fig. 9. Packets blocked in the $y$ direction: (a) south-to-north and (b) north-to-south direction.

1. Default Routing,

2. Single OIP,

3. Multiple OIPs, and

4. f-chain.

If no OIP blocks the routing paths in the Default Routing, packets are routed following Default Routing. Otherwise, packets are routed following Single OIP, Multiple OIPs, or f-chain to detour OIPs. The whole OAPR algorithm is introduced from Default Routing to f-chain in Sections 3.1, 3.2, 3.3, and 3.4.

\subsection{Default Routing}

The Default Routing is YX routing based on the odd-even turn model. When no OIP blocks the routing path, a packet is routed along the $y$ dimension first until the $y$ coordinate is the same as the destination. Then, the packet is routed along the $x$ dimension until the destination. The possible cases in the Default Routing are shown in Fig. 8. The symbol $E(O)$ means that packets turn at even (odd) columns. The Default Routing in the OAPR contains the turns at odd columns, which are not included in the E-XY. Therefore, the OAPR can balance traffic loads between odd and even columns.

\subsection{Single OIP}

In Section 3.2, the routing paths of Default Routing blocked by a single OIP are discussed. How the OAPR can achieve balanced traffic loads and shorter routing paths is shown. We divide Single OIP into two parts: 1) packets blocked in the $y$ direction and 2) packets blocked in the $x$ direction.

\subsubsection{Packets Blocked in the $y$ Direction}

Packets blocked in the $y$ direction can be analyzed according to the locations of sources and destinations. Possible situations are shown in Fig. 9. The symbols $x_{m-1}$, $y_{m}, y_{M}$, and $O$ stand for the column $x_{m}-1$, the row $y_{m}$, the row $y_{M}$, and the nearest odd column in the east direction (column $x_{M}+1$ or $x_{M}+2$ ) if an OIP is located at $\left[x_{m}, x_{M}, y_{m}, y_{M}\right]\left(x_{m} \leq x_{M}\right.$ and $y_{m} \leq y_{M}$, where $x_{m}, x_{M}, y_{m}$, and $y_{M} \in\{0,1, \ldots, n-1\}$ in an $n \times n$ mesh). The routers with oblique lines cannot be connected to normal-sized IPs in the OAPR. For packets transmitted from regions $I$ to $I I I$, the packets move westward first and turn at column $x_{m}-1$. Then, the packets go northward along column $x_{m}-1$ until the $y$ coordinate is the same as the destination. After that, the packets go eastward until the destination. The 


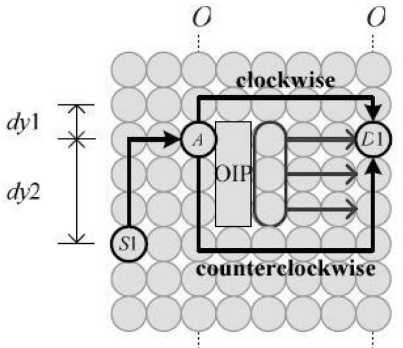

(a) (b)

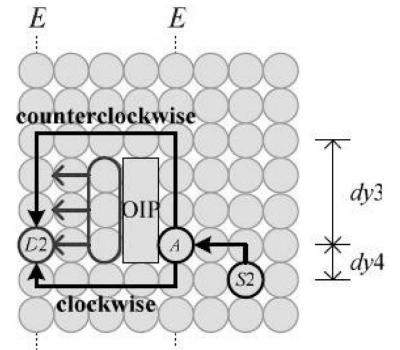

Fig. 10. Packets blocked in the $x$ direction: (a) west-to-east and (b) eastto-west direction.

source-destination pair S1-D1 (Fig. 9a) shows a simple example. For packets transmitted from regions $I$ to $I$, the packets move along the $x$ direction until the $x$ coordinate is the same as the destination. Then, the packets go northward to the destination. The packets transmitted from regions $I$ to $I V$ are similar to the case from regions $I$ to $I I$, but these packets can only turn at odd columns. The sourcedestination pairs $S 2-D 2$ and $S 3-D 3$ show two examples. Additionally, the paths from regions $I$ to $I V$ are shorter than the paths in the E-XY because the turns are unused in the E-XY, as shown in Figs. 6a and 6b. Therefore, these paths alleviate the loads in the north, south, east, and west boundaries of the OIP, as shown in Figs. 9a and 9b.

\subsubsection{Packets Blocked in the $x$ Direction}

If packets are blocked in the $x$ direction, the OAPR selects the paths shown in Figs. 10a and 10b. When the packets collide with the OIPs at router $A$ in Figs. 10a and 10b, the packets select the clockwise or counterclockwise path to dodge the OIPs until the destination $D 1$ and $D 2$ are reached. In order to shorten the paths, $d y 1-d y 4$ must be concerned to select the better choice. If $d y 1<d y 2(d y 3<d y 4)$, the clockwise (counterclockwise) path is selected. Otherwise, the counterclockwise (clockwise) path is chosen. The routing paths reduce the traffic loads at the east or west boundaries of OIPs if packets are blocked in the east-to-west or west-toeast direction, as shown in Figs. 10a and 10b.

Besides, two special cases are shown in Figs. 11a and $11 \mathrm{~b}$. If packets are routed at the corners of the OIPs (the routers with oblique lines), dotted lines are selected by the

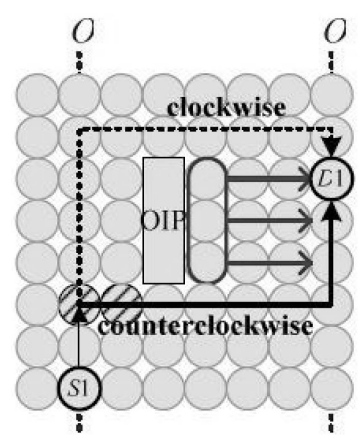

(a)

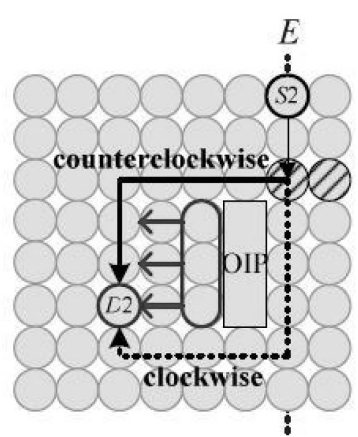

(b)
Fig. 11. Special cases for the packets blocked in the $x$ direction: (a) northeast and (b) southwest direction.

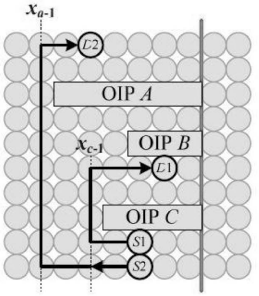

(a)

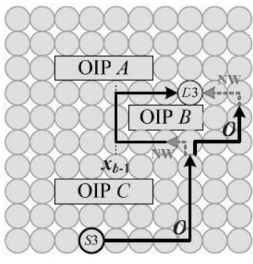

(b)

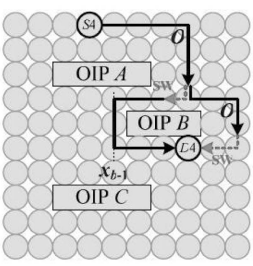

(c)
Fig. 12. Three cases of OIPs placed vertically: (a) OIPs are aligned on the east edge. Invalid (b) NW and (c) SW turns happen if OIPs are not aligned on the east edge.

rules discussed above. Obviously, the dotted lines have longer distances. Therefore, the OAPR selects the paths along the solid lines. Two symmetric cases (northwest and southeast direction) are not shown in Fig. 11.

\subsection{Multiple OIPs}

In this section, the routing paths of Default Routing blocked by multiple OIPs are discussed. Two cases are considered: 1) OIPs placed vertically and 2) OIPs placed horizontally.

\subsubsection{OIPs Placed Vertically}

Fig. 12 shows the case of OIPs placed vertically. Although the condition seems more complicated than single OIP, the routing rules described in Section 3.2.1 are still workable. In Fig. 12a, two cases ( $S 1$ to $D 1$ and $S 2$ to $D 2$ ) follow the same routing rules from regions $I$ to III in Fig. 9a. The symbols $x_{c-1}$ and $x_{a-1}$ stand for columns $x_{c}-1$ and $x_{a}-1$ if the OIPs $A$ and $C$ are placed at $\left[x_{a}, x_{A}, y_{a}, y_{A}\right]$ and $\left[x_{c}, x_{C}, y_{c}, y_{C}\right]$ $\left(x_{a} \leq x_{A}, y_{a} \leq, y_{A}, x_{c} \leq x_{C}\right.$, and $y_{c} \leq y_{C}$, where $x_{a}, x_{A}, y_{a}$, $y_{A}, x_{c}, x_{C}, y_{c}$, and $y_{C} \in\{0,1, \ldots, n-1\}$ in an $n \times n$ mesh). First, the packets from $S 1$ to $D 1$ (S2 to $D 2$ ) are routed westward until column $x_{c}-1\left(x_{a}-1\right)$ is met. After that, the packets move northward and then go eastward until destination $D 1(D 2)$.

Besides, all OIPs overlapping vertically must be aligned on the east edge, as shown in Fig. 12a. This is possible because the OIP placements are controllable. Without this restriction, some kinds of placements cause the OAPR to fail. Two examples are shown in Figs. $12 \mathrm{~b}$ and $12 \mathrm{c}$. If OIP $B$ is not aligned on the east edge, no packets can be transmitted from $S 3$ to $D 3$ and $S 4$ to D4. The NW and SW turns at the odd columns cause the paths to avoid the oddeven turn model.

\subsubsection{OIPs Placed Horizontally}

If multiple OIPs are placed horizontally, the previous routing rules described in Section 3.2.2 are still workable, as shown in two examples (S1 to D1 and $S 2$ to $D 2$ ) in Fig. 13. The packets from $S 1$ (S2) are routed to destination $D 1$ (D2) successfully without any new rules.

However, the special cases in Fig. 14 may occur. The packets following the routing rules from regions $I$ to $I I$ and $I$ to $I V$ in Fig. 9 are blocked by other OIPs, shown as dotted lines. Therefore, the packets from $S 1$ (S2) must turn at the odd (even) column and go along the solid lines until destination $D 1(D 2)$. This is a new rule for the routings in 


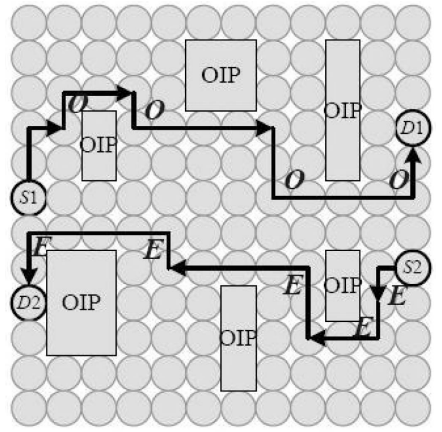

Fig. 13. Routing paths of multiple OIPs placed horizontally.

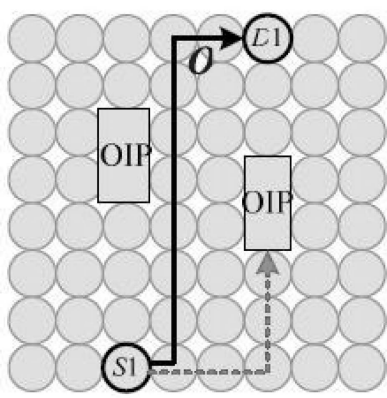

(a)

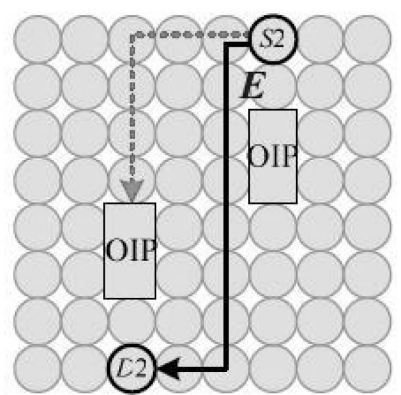

(b)
Fig. 14. Special cases for the packets blocked by other OIP: (a) northeast and (b) southwest direction.

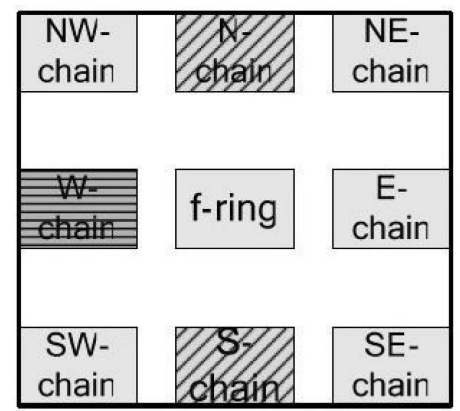

Fig. 15. The routing modes of the OAPR for $\mathrm{f}$-rings and eight different kinds of $f$-chains.

this mode (Multiple OIPs). Two symmetric cases (northwest and southeast direction) are not shown in Fig. 14.

\section{4 f-Chain}

The routing rules of $\mathrm{f}$-rings are presented in the previous sections. Fig. 15 shows an f-ring and eight types of f-chains discussed in Section 1. In the OAPR, the routing rules of f-rings can be directly applied to NW-chains, NE-chains, E-chains, SW-chains, and SE-chains without any modification. For N-chains and S-chains, only a few rules need to be modified. However, there are big problems on $\mathrm{W}$-chains because the paths detouring $\mathrm{W}$-chains must contain invalid turns in the odd-even turn model. Hence, the OAPR prevents deadlock by restricting some OIP placements.

\subsubsection{N-Chain and S-Chain}

When packets are routed in the $x$ direction and blocked by an N-chain, as shown in Fig. 16, the packets should originally follow the paths shown in Figs. 10 or 11. It

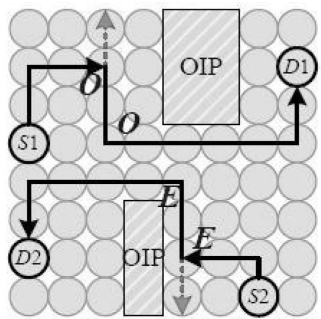

Fig. 16. The routing paths of the OAPR for an N-chain and an S-chain.

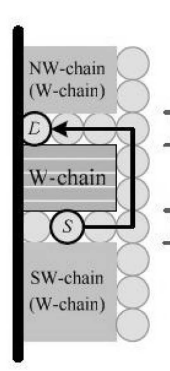

(a)

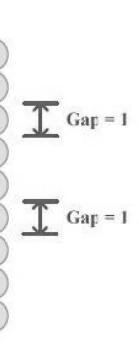

(b)

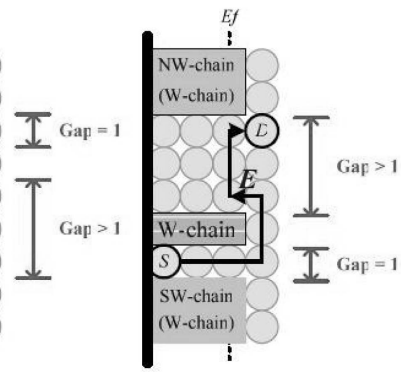

(c)
Fig. 17. Three cases to detour W-chains: (a) two gap $=1$, (b) upper gap $=1$ and lower gap $>1$, and (c) upper gap $>1$ and lower gap $=1$.

means that the paths with dotted lines in Fig. 16 could happen. The packets bump into the north or the south boundary of the irregular mesh and the routing mechanism fails because 180-degree turns are prohibited in the oddeven turn model. Therefore, the OAPR forces the packets from $S 1$ to be routed along the solid line until destination $D 1$. A similar situation of an S-chain ( $S 2$ to D2) is also shown in Fig. 16.

\subsubsection{W-Chain}

The routing rules of $\mathrm{W}$-chains are big problems because of the odd-even turn model. Fig. 17 shows three cases of detouring W-chains. In Fig. 17a, the packet cannot move westward as f-rings because of the west boundary of the irregular mesh. Hence, the only way is to route the packet around the $\mathrm{W}$-chain to the destination, shown as the solid line. In Fig. 17b, the packet goes northward to the boundary of the W-chain and then follows the path in Fig. 17a. The case in Fig. 17c is opposite to Fig. 17b. First, the packet is routed around the $\mathrm{W}$-chain until the first even column $\left(E_{f}\right)$. After that, the packet turns at column $E_{f}$ to avoid lengthening the path. Finally, the packets go northward and then eastward (or westward in another case) to destination $D$.

However, these paths may cause deadlock because of the invalid turns in the odd-even turn model. Fig. 18 shows two possible cases in which deadlock occurs. Two loops ( $\{L 1, L 2\}$ and $\{L 3, L 4\})$ and the invalid turns (dotted lines) result in deadlock. Therefore, we restrict the OIP placements to prevent deadlock: There is only one gap greater than 1 in Fig. 17. This restriction breaks one of the two loops $\{L 1, L 2\}$ and $\{L 3, L 4\}$ in Fig. 18. Hence, deadlock can be avoided because the odd-even model does not allow 180-degree turns.

We have introduced the whole routing paths under different placements. Our OAPR routing can make traffic 


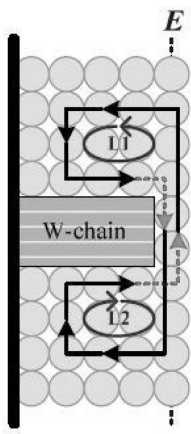

(a)

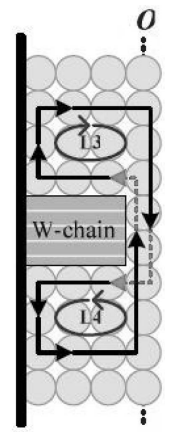

(b)
Fig. 18. Deadlock occurs on (a) even and (b) odd columns when detouring W-chains.

more balanced and have shorter paths. However, there are some placement restrictions, which our algorithm cannot support due to some inevitable reasons.

\subsection{Restrictions on OIP Placements}

When we use the OAPR, there are several placement rules:

1. For an OIP located at $\left[x_{m}, x_{M}, y_{m}, y_{M}\right]\left(x_{m} \leq x_{M}\right.$ and $y_{m} \leq y_{M}$, where $x_{m}, x_{M}, y_{m}$, and $y_{M} \in$ $\{0,1, \ldots, n-1\}$ in an $\mathrm{n} \times \mathrm{n}$ mesh), the routers at range $\left[x_{m}-2, x_{M}+2, y_{m}-1, y_{M}+1\right]$ can be only linked to normal-sized OIPs. These routers are reserved to satisfy the routings based on the oddeven turn model, which is similar to the E-XY [10].

2. The routers on the east side of an OIP cannot be connected to normal-sized IPs. This rule also appears in the E-XY [10].

3. All OIPs vertically overlapping must be aligned on the east edge.

4. At most one gap can be greater than 1 at the west boundary of irregular meshes.

Fig. 19 shows an example with rules 1-4 described above. Rules 1 and 2 are the same as the E-XY [10] due to the restriction of the routings based on the odd-even turn model. Rules 3 and 4 can prevent deadlock in the networks using the OAPR. Because the designers can control the OIP placements in irregular meshes, it is possible to follow rules 1-4. As long as the rules are followed, the OAPR works correctly and makes the networks perform better.

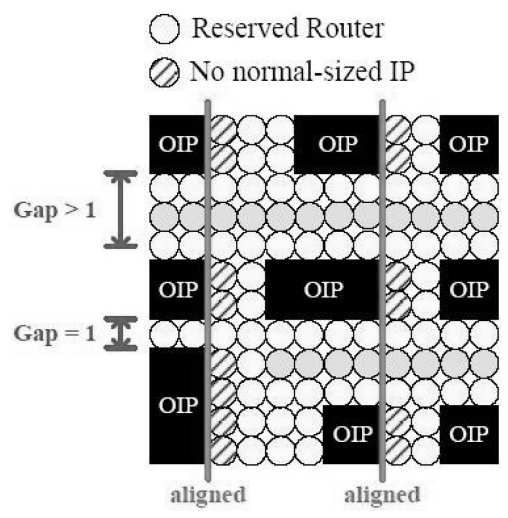

Fig. 19. Restrictions on OIP placements for the OAPR.
TABLE 1

Procedure to Find the STLD

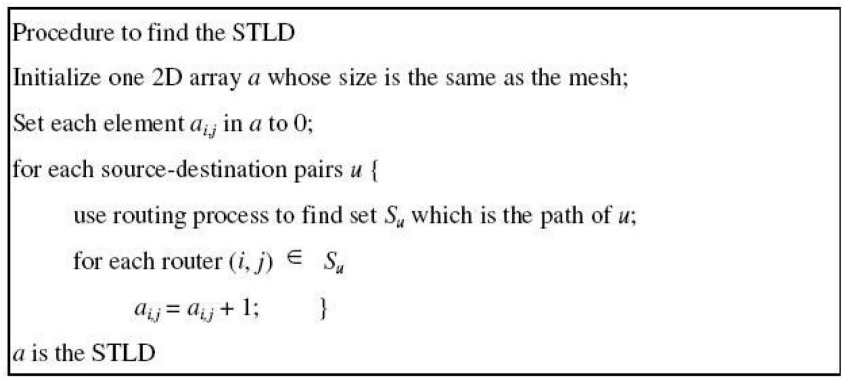

\section{Performance Evaluation}

To compare the performance of Chen and Chiu's [8], the E-XY [10], and the OAPR, three statistical cases (Cases 1, 2, and 3) are simulated according to different OIP placements. Cases 1, 2, and 3 correspond to Single OIP, Multiple OIPs, and f-chain, respectively, as discussed in Section 3. In addition, a real application is utilized to evaluate the OAPR in Section 4.4.

We introduce two methods, 1) static analysis and 2) dynamic simulation, to evaluate the performance of the OAPR for each case. Static analysis uses statistical information to evaluate network performance quickly. Dynamic simulation runs cycle-accurate simulation on a SystemC simulator. After that, we introduce three traffic patterns: 1) random, 2) exponential, and 3) Rent's rule [16] to model different applications in practice. Finally, the simulation results show that the networks using the OAPR perform better than those using the E-XY [10] and Chen and Chiu's [8].

\subsection{Case 1: Traffic of Single OIP}

\subsubsection{Static Analysis}

Statistical traffic load distribution (STLD) helps us to predict the traffic load of the network statistically without real experiments. According to different routing algorithms, the STLD collects all source-destination pairs to estimate real simulations with uniform traffic patterns. Let the packets of all source-destination pairs route once and statistically count the number of packets passing through in each router. The result forms a $2 \mathrm{D}$ distribution graph, which stands for the STLD. The detailed procedure is described in Table 1.

The STLD represents the number of packets of all source-destination pairs passing through the corresponding routers. According to the distribution, we can predict whether the traffic loads of a network are balanced or not and the regions where the contention might be more serious. Moreover, we can use the average hop count $\left(H_{\text {avg }}\right)$ of packets and the standard deviation $(\sigma)$ between $a_{i, j}$ in $a$ to estimate the network performance. The details are shown below:

- $\sigma$ : standard deviation of the load of $\left\{R_{N I}, R_{W I}\right\}$.

- $H_{\text {avg }}$ : summation $(a) /$ combination $\left(R_{W I}\right)$.

The reserved routers in Fig. 19 are defined as $R_{N I}$. The routers connected to either normal-sized IP or OIP are defined as $R_{W I}$. 


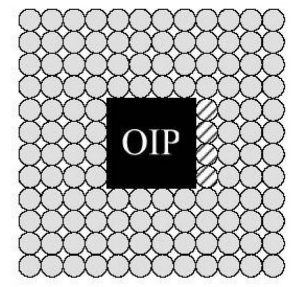

(a)

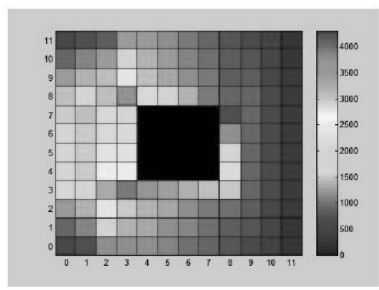

(c)

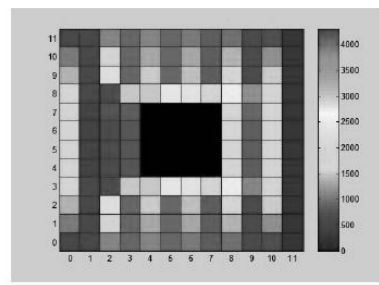

(b)

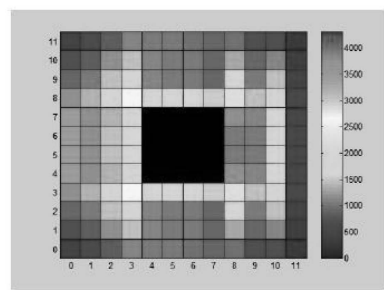

(d)
Fig. 20. Case 1: (a) a single OIP in a $12 \times 12$ irregular mesh and the STLDs of (b) the E-XY, (c) Chen and Chiu's, and (d) the OAPR.

The static analysis of a single OIP in a $12 \times 12$ irregular mesh is shown in Fig. 20a. The STLDs of the E-XY, Chen and Chiu's, and the OAPR are shown in Figs. 20b, 20c, and $20 \mathrm{~d}$. The color bar represents the range of the statistical traffic loads. In addition, black blocks in STLDs mean the location of the OIP and other colored blocks represent the statistical traffic loads at the corresponding routers in Fig. 20a.

In Fig. 20b, there are dense loads on the west side of the OIP. Moreover, stripes with deep and light color on the whole network mean that the statistical loads on the odd and even columns are unbalanced. In addition, the STLD of Chen and Chiu's still concentrates the loads around the OIP in Fig. 20c. In contrast, the OAPR results in balanced statistical traffic loads not only around the OIP but also on the whole network in Fig. 20d. Besides, according to $H_{\text {avg }}$ and $\sigma$ shown in Table 2, the OAPR causes shorter routing paths and balanced statistical traffic loads in the irregular mesh.

\subsubsection{Dynamic Simulation}

To evaluate the realistic performance of the proposed algorithm, we have developed a simulator in SystemC language. The network environment is assumed as a 2D mesh with wormhole switching mechanism. Two unidirectional channels exist between each pair of neighboring routers. Each channel of a router has an input queuing buffer with size of two flits and round-robin is the policy of

TABLE 2

The Results of the STLDs

\begin{tabular}{|c|c|c|c|c|c|c|}
\hline & \multicolumn{2}{|c|}{ Case I } & \multicolumn{2}{c|}{ Case II } & \multicolumn{2}{c|}{ Case III } \\
\cline { 2 - 7 } & $H_{\text {avg }}$ & $\sigma$ of load & $H_{\text {avg }}$ & $\sigma$ of load & $H_{\text {avg }}$ & $\sigma$ of load \\
\hline E-XY [10] & 10.09 & 923 & 10.45 & 717 & $\mathrm{X}$ & $\mathrm{X}$ \\
\hline Chen and Chiu's [8] & 9.84 & 815 & 10.41 & 714 & 7.49 & 446 \\
\hline OAPR & 8.85 & 448 & 8.73 & 414 & 7.26 & 416 \\
\hline
\end{tabular}

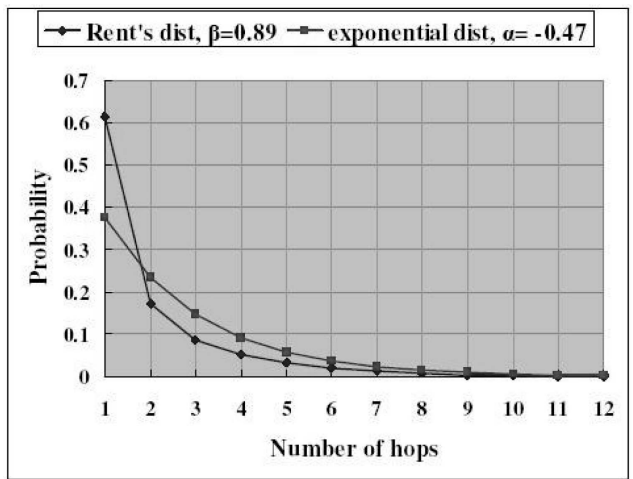

Fig. 21. Traffic distribution of Rent's rule and the exponential traffic patterns.

arbitration. We assume that one flit passing through one router takes one cycle. IPs are connected to each router except for the restricted routers. IPs generate packets at time intervals chosen from a negative exponential distribution. For simplicity, we assume that each packet contains five flits. For each run of the simulation, we collect 50,000 generated packets and discard the first 10,000 warm-up packets. After the simulation, we obtain an average latency versus injection rate.

Three traffic patterns, random, exponential, and Rent's rule, are introduced as follows: In the random traffic pattern, an IP sends a packet to any other routers with equal probability. The random (Rand) traffic pattern is a simple way to estimate performance. In [16], the exponential (Exp) and Rent's rule (Rent) traffic patterns are utilized to model the first hop accounts for the bulk of OCN traffic. It means that communication between neighboring routers is the primary contributor to OCN congestion and power consumption. Fig. 21 shows the distributions of the Rent's rule and the exponential traffic patterns. Our experiments are simulated according to these traffic patterns.

The latency versus injection rate of Case 1 is shown in Fig. 22. The OAPR performs better because of the more balanced traffic loads and the shorter paths. Therefore, the network can support more traffic by using the OAPR and that is the reason why the latency of the OAPR ascends latter. The results of SystemC simulation correspond to the results of the STLD. Besides, it is proven that the STLD is helpful to estimate the performance of irregular meshes using different routing algorithms.

The detailed information in Fig. 22 is as described in the following: When the injection rate is low, the OAPR can keep the latency lower than Chen and Chiu's [8] and E-XY [10] because of less $H_{\text {avg }}$. When the injection rate is getting higher, the OAPR can keep contention lower because of more balanced traffic loads. Therefore, the latency of OAPR is lower than Chen and Chiu's [8] and E-XY [10]. In the random traffic pattern, the networks using Chen and Chiu's [8], the E-XY [10], and the OAPR saturate at the injection rate of $0.03,0.035$, and 0.06 flits/IP/cycle. These give 71.4 percent and 100 percent improvements to Chen and Chiu's [8] and E-XY [10] in terms of sustainable throughput. 


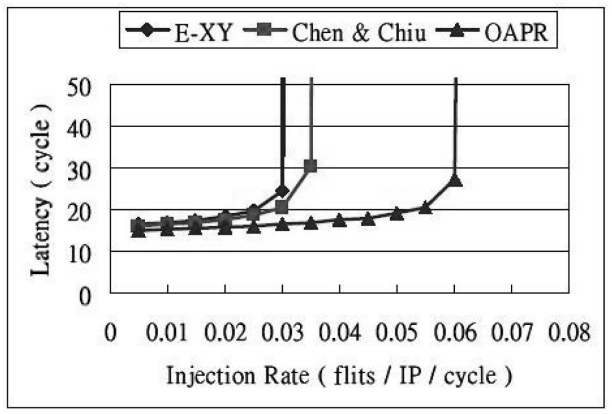

(a)

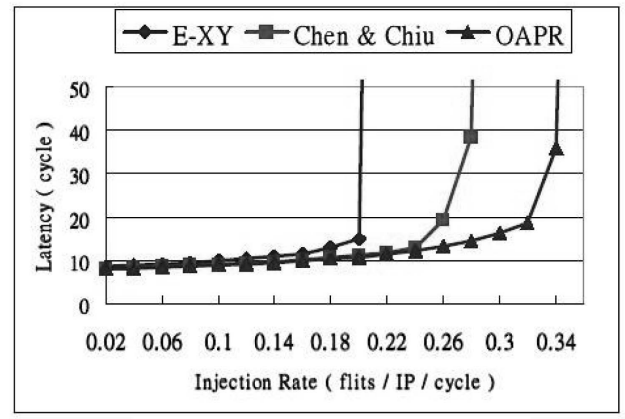

(b)

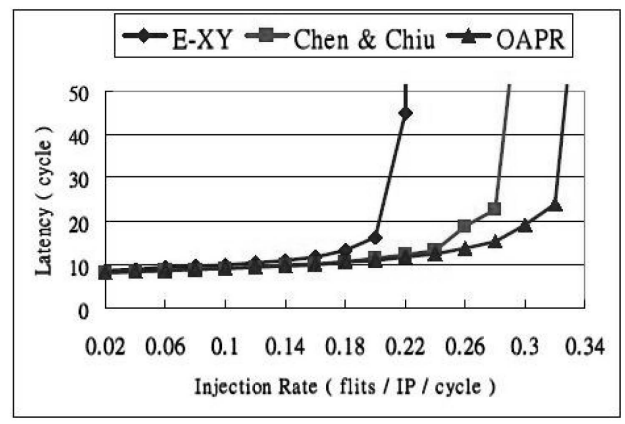

(c)

Fig. 22. Case 1: The latency versus injection rate of the OAPR, Chen and Chiu's [8], and the E-XY [10] with (a) the random, (b) Rent's rule, and (c) the exponential traffic patterns.

In the Rent and the Exp traffic patterns, the networks saturate at a higher injection rate than the Rand traffic pattern. However, the improvement is less than the Rand traffic pattern because lots of packets are transmitted to neighbor routers. The improvements to Chen and Chiu's [8] and the E-XY [10] are 13.3 percent to 70.0 percent in terms of sustainable throughput. The performance improvement of throughput is summarized in Table 3. The networks using the OAPR still perform better than those using the E-XY and Chen and Chiu's in the Rent and the Exp traffic patterns.

\subsection{Case 2: Traffic of Multiple OIPs}

To verify the functionality and performance in the case of Multiple OIPs, we place four OIPs as shown in Fig. 23a. In this case, the STLDs are shown in Figs. 23b, 23c, and 23d. We can observe that the E-XY [10] and Chen and Chiu's [8] make the statistical traffic loads unbalanced, while the OAPR does not. According to Table 2 and Fig. 23, all trends in Case 2 are similar to Case 1. In addition, the latency
TABLE 3

Summaries of Throughput Improvement to Chen and Chiu's [8] and the E-XY [10]

\begin{tabular}{|c|c|c|c|c|c|c|}
\hline & \multicolumn{3}{|c|}{ Chen and Chiu's [8] } & \multicolumn{3}{c|}{ E-XY [10] } \\
\cline { 2 - 7 } & Random. & Rent & Exp & Random & Rent & Exp \\
\hline Case I & $71.4 \%$ & $13.3 \%$ & $13.3 \%$ & $100.0 \%$ & $70.0 \%$ & $54.5 \%$ \\
\hline Case II & $71.4 \%$ & $33.3 \%$ & $33.3 \%$ & $71.4 \%$ & $77.8 \%$ & $77.8 \%$ \\
\hline Case III & $55.6 \%$ & $15.4 \%$ & $15.4 \%$ & $\mathrm{X}$ & $\mathrm{X}$ & $\mathrm{X}$ \\
\hline
\end{tabular}

versus injection rate is shown in Fig. 24. The results show that the networks using OAPR perform better than those using the E-XY [10] and Chen and Chiu's [8] in the Rand, Rent, and Exp traffic patterns. The improvement to Chen and Chiu's [8] and the E-XY [10] are 33.3 percent to 77.8 percent in terms of sustainable throughput, which is summarized in Table 3. It is proven that, no matter where we place a single OIP or multiple ones, the OAPR performs better.

\subsection{Case 3: Traffic of an f-Ring and f-Chains}

The two cases above only contain f-rings. (No OIP adjoins the boundaries of meshes.) Now, we place eight kinds of f-chains and an f-ring to verify the entire algorithms. The placements are shown in Fig. 25a. The placements of $\mathrm{W}$-chains and SW-chains follow the rules discussed in Section 3.5. In this case, the E-XY [10] is not included due to its own restriction, which is described in Section 2.3. According to Table 2 and Fig. 25, all trends are alike. The latency versus injection rate is shown in Fig. 26. The results show that the networks using OAPR perform better than those using Chen and Chiu's [8] in the Rand, Rent, and Exp traffic patterns. The improvement is 15.4 percent to 55.6 percent in terms of sustainable throughput, which is summarized in Table 3.

In summary, we examine three cases of placement for the E-XY [10], Chen and Chiu's [8], and the OAPR. The network using the OAPR has fewer average hop counts and balanced traffic loads. Therefore, the latency could be lower

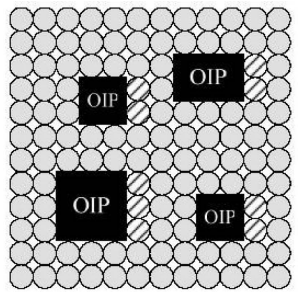

(a)

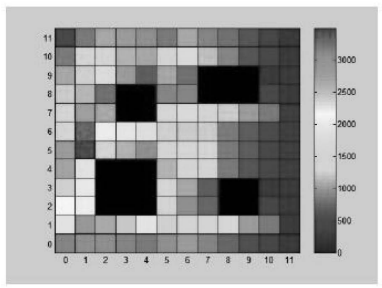

(c)

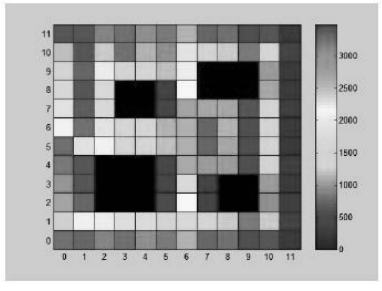

(b)

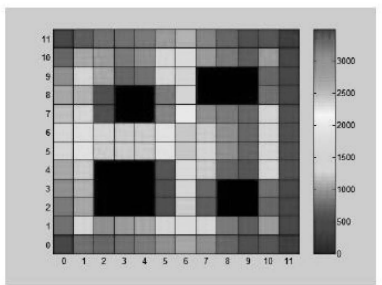

(d)
Fig. 23. Case 2: (a) Multiple OIPs and the STLDs of (b) the E-XY [10], (c) Chen and Chiu's [8], and (d) the OAPR. 


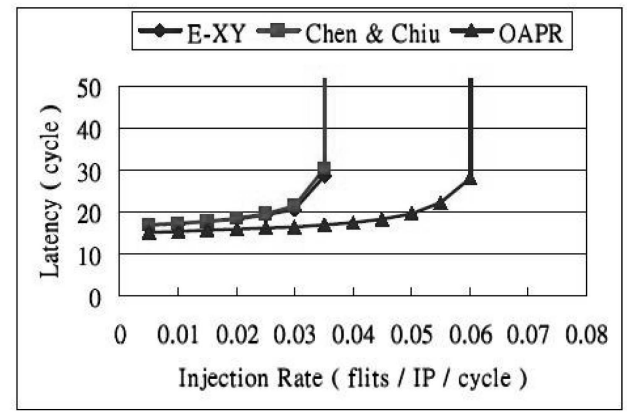

(a)

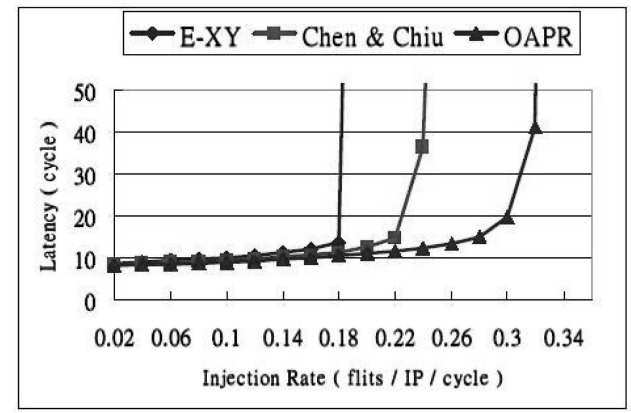

(b)

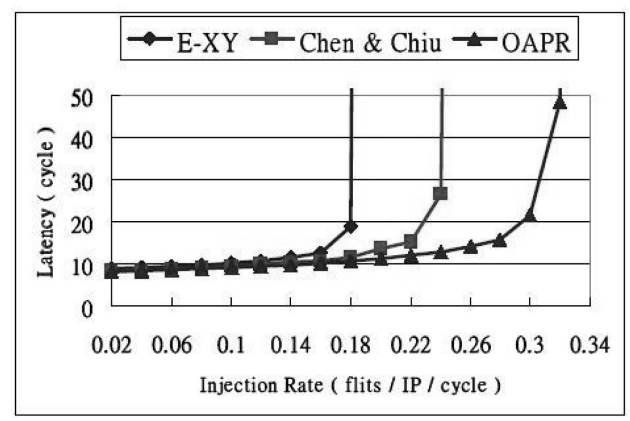

(c)

Fig. 24. Case 2: The latency versus injection rate of the OAPR, Chen and Chiu's algorithm [8], and the E-XY [10] with (a) the random, (b) Rent's rule, and (c) the exponential traffic patterns.

and the performance is better. Moreover, the E-XY only supports f-rings, while Chen and Chiu's and the OAPR can support not only f-rings but also f-chains.

\subsection{A Realistic Simulation: A Multimedia System}

In this section, a real application (a MultiMedia System) with real traffic patterns is utilized to evaluate the performance of the E-XY [10], Chen and Chiu's [8], and the OAPR. The MultiMedia System (MMS) is an integrated video/audio system. The MMS includes an h263 video encoder, an h263 video decoder, an mp3 audio encoder, and an mp3 audio decoder. In [18], the MMS was partitioned into 40 distinct tasks and mapped to 25 IPs. Fig. 27a shows the communication task graph. The communication volumes between IPs are not shown. If some of the IPs are oversized, the mapping in [18] is unworkable. In our simulation, IP 6 (an embedded DRAM) is assumed to be an OIP and the MMS is mapped to a $6 \times 6$ irregular mesh, as shown in Fig. 27b. IP 6 can communicate with other IPs through the routers in $(1,2)$ and $(1,4)$. In

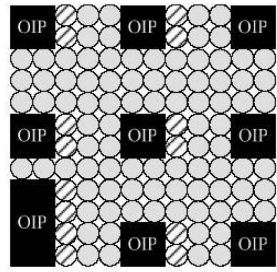

(a)

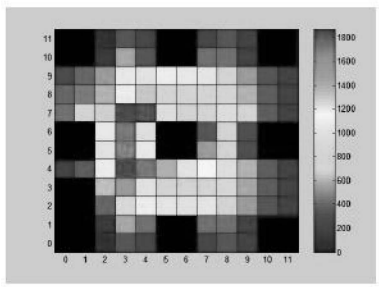

(b)

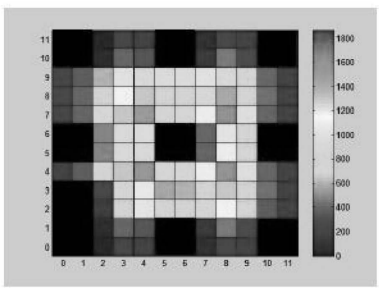

(c)
Fig. 25. Case 3: (a) f-ring and f-chains and the STLDs of (b) Chen and Chiu's [8] and (c) the OAPR.

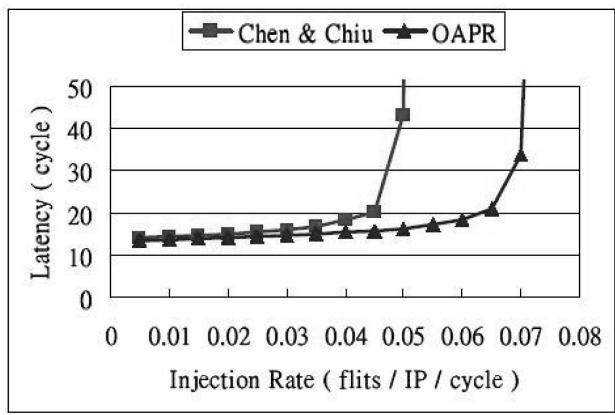

(a)

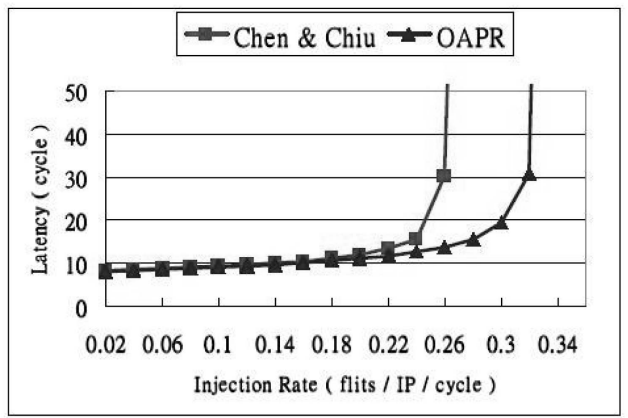

(b)

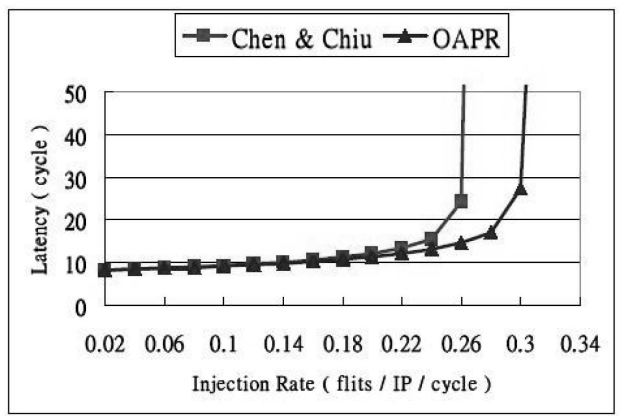

(c)

Fig. 26. Case 3: The latency versus injection rate of the OAPR and Chen and Chiu's algorithm [8] with (a) the random, (b) Rent's rule, and (c) the exponential traffic patterns. 


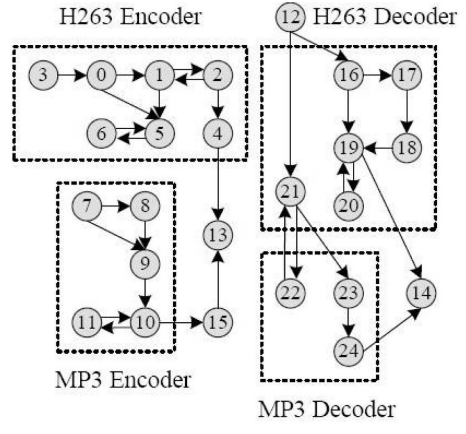

(a)

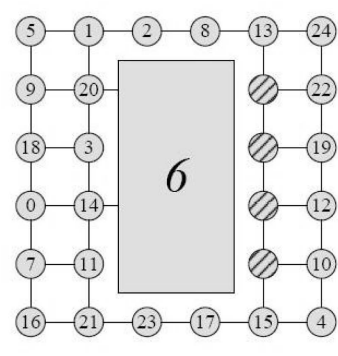

(b)
Fig. 27. A MultiMedia System: (a) the communication task graph and (b) a possible mapping.

general, the IP mapping usually strongly depends on the target OCN applications. To have intuitive and fair comparisons among all routing algorithms in irregular meshes, the locations of normal-sized IPs are determined by a random IP mapping algorithm in our experiment. That is, we run the random mapping 1,000 times and sort the results by the communication cost function from that in [21]. Then, we select the median case as a design example in our simulation, as shown in Fig. 27b.

The latency versus injection rate is shown in Fig. 28. The improvement is 20 percent to 55.6 percent in terms of sustainable throughput. The results show that the networks using OAPR still perform better than those using the E-XY [10] and Chen and Chiu's [8] in the MMS.

\section{Hardware Overhead of the OAPR}

In this section, the hardware implementation of the OAPR is introduced. Because the OAPR is deterministic routing, we can implement the OAPR by lookup tables (LUTs) [15]. Fig. 29a shows the basic 5-port router model. Each port has a corresponding routing logic and each routing logic keeps the information of destination addresses (Addr.) and output directions (Out) in LUTs. In routing process, the output direction is selected according to different destination addresses. In Fig. 29b, the OAPR design flow is proposed

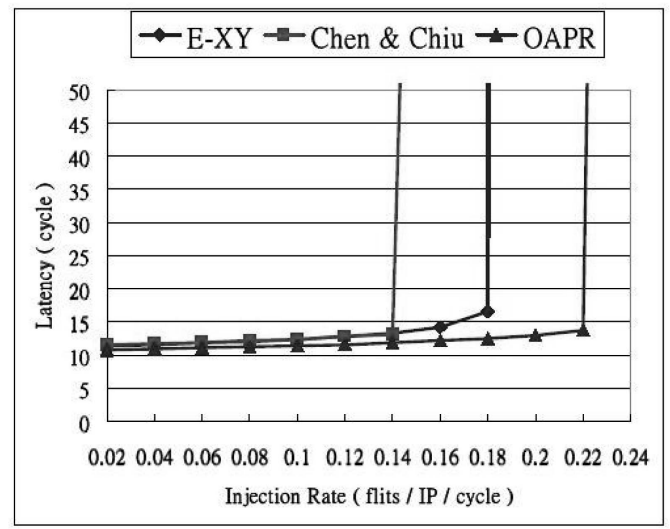

(a)

(b)

Fig. 28. The latency versus injection rate of the OAPR, Chen and Chiu's algorithm [8], and the E-XY [10] in the MultiMedia System.

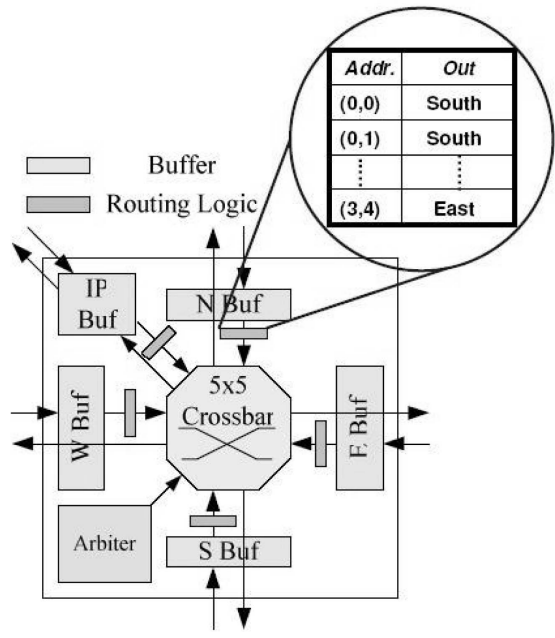

(a)

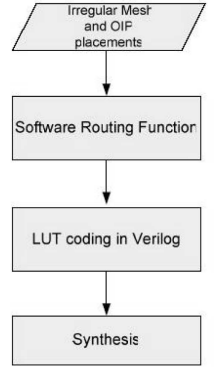

(b)

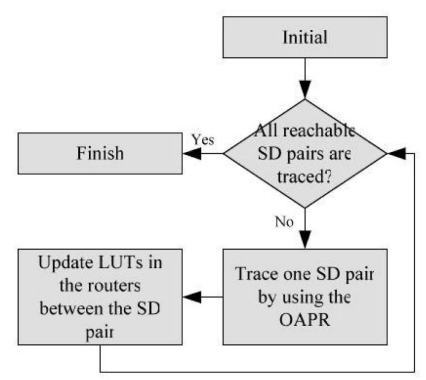

(c)
Fig. 29. The OAPR design flow: (a) the routing logic in the 5-port router model, (b) the flowchart of the OAPR design flow, and (c) the flowchart to update LUTs.

to implement the routing logic. The input is an irregular mesh with OIP placements from EDA tools. The OAPR routing design tool is a software tool to determine the Addr. and Out in the LUTs and generate RTL codes automatically. The detailed executions of this flow are described as follows:

1. Software routing function. First, the OAPR routing design tool is executed to determine the Addr. and Out in the LUTs. Fig. 29c shows the flowchart to update LUTs. All reachable source-destination pairs are traced in irregular meshes. The path between each source-destination pair is routed once by using the OAPR. In each router, the routing information is recorded by the LUTs in each router if packets pass through. After this phase, all LUTs are obtained. According to the Addr. and Out in the LUTs, the packets can be routed following the OAPR.

2. LUT coding in Verilog. In this phase, the OAPR routing design tool can generate the synthesizable RTL code. The Addr. and Out in Step 1 are utilized to generate RTL code of each routing logic automatically.

3. Synthesis. Finally, the RTL codes are handed over to the synthesis tool.

The generated codes are synthesized by Synopsys Design Vision and the technology is TSMC $0.18 \mu \mathrm{m}$ CMOS. The 
Cases 1, 2, and 3 described in Sections 4.1, 4.2, and 4.3 are implemented. The average gate counts of a routing logic in three cases are 14.2, 24.9, and 24.5. Finally, we compare the area of routing logics with a whole router implemented in [14]. The area of the whole router is around 11,000 gates. The overhead of routing logics is less than 1 percent in three cases. This proves that the routing algorithm is feasible for OCN.

\section{Conclusions}

In this paper, we propose a new routing algorithm, OAPR, based on the odd-even turn model for irregular mesh-based OCNs. Due to the turn model, virtual channels are not needed to solve deadlock. Besides, traffic loads on the boundaries of OIPs can be alleviated and the loads of the network are evenly dispersed. The experiments prove that the OAPR has lower latency and gives 13.3 percent to 100 percent improvement compared to the other two faulttolerant algorithms. Moreover, implementing the algorithm is feasible because of the negligible overhead (less than 1 percent). In conclusion, the OAPR has better performance and is feasible for OCNs.

\section{ACKNOWLEDGMENTS}

This work was supported by the National Science Council under NSC Grant 96-2221-E-002-243.

\section{REFERENCES}

[1] ITRS, Int'1 Technology Roadmap for Semiconductors, http:// public.itrs.net, 2008.

[2] J.A. Davis et al., "Interconnect Limits on Gigascale Integration (GSI) in the 21st Century," Proc. IEEE, vol. 89, pp. 305-324, Mar. 2001.

[3] R. Ho, K.W. Mai, and M.A. Horowitz, "The Future of Wires," Proc. IEEE, vol. 89, pp. 490-504, Apr. 2001.

[4] D. Sylvester and K. Keutzer, "A Global Wiring Paradigm for Deep Submicron Design," IEEE Trans. CAD of Integrated Circuits and Systems, vol. 19, pp. 242-252, Feb. 2000.

[5] S. Kumar et al., "A Network on Chip Architecture and Design Methodology," Proc. Int'l Symp. Very Large Scale Integration, pp. 105-112, 2002.

[6] E. Bolotin, I. Cidon, R. Ginosar, and A. Kolodny, “QNoC: QoS Architecture and Design Process for Network on Chip," J. Systems Architecture, vol. 50, pp. 105-128, Feb. 2004.

[7] M.K.F Schafer, T. Hollstein, H. Zimmer, and M. Glesner, "Deadlock-Free Routing and Component Placement for Irregular MeshBased Networks-on-Chip," Proc. IEEE/ACM Int'l Conf. ComputerAided Design, pp. 238-245, 2005.

[8] K.-H. Chen and G.-M. Chiu, "Fault-Tolerant Routing Algorithm for Meshes without Using Virtual Channels," J. Information Science and Eng., vol. 14, pp. 765-783, Dec. 1998.

[9] R.V. Boppana and S. Chalasani, "Fault-Tolerant Wormhole Routing Algorithms for Mesh Networks," IEEE Trans. Computers, vol. 44, pp. 848-864, 1995.

[10] J. Wu, "A Fault-Tolerant and Deadlock-Free Routing Protocol in 2D Meshes Based on Odd-Even Turn Model," IEEE Trans. Computers, vol. 52, no. 9, pp. 1154-1169, Sept. 2003.

[11] R. Holsmark and S. Kumar, "Design Issues and Performance Evaluation of Mesh NoC with Regions," Proc. Norchip Conf., pp. 40-43, 2005.

[12] G.J. Glass and L.M. Ni, "The Turn Model for Adaptive Routing," J. ACM, vol. 40, pp. 874-902, Sept. 1994.

[13] G.M. Chiu, "The Odd-Even Turn Model for Adaptive Routing," IEEE Trans. Parallel and Distributed Systems, vol. 11, no. 7, pp. 729737, July 2000.
[14] J. Hu and R. Marculescu, "DyAD—Smart Routing for Networkson-Chip," Proc. Design Automation Conf. (DAC '04), pp. 260-263, June 2004.

[15] J. Hu and R. Marculescu, "Energy- and Performance-Aware Mapping for Regular NoC Architecture," IEEE Trans. ComputerAided Design of Integrated and Systems, vol. 24, pp. 551-562, Apr. 2005.

[16] D. Greenfield, A. Banerjee, J.-G. Lee, and S. Moore, "Implications of Rent's Rule for NoC Design and Its Fault-Tolerance," Proc. ACM/IEEE Int'l Symp. Networks-on-Chip, pp. 283-294, May 2007.

[17] R. Holsmark and S. Kumar, "Corrections to Chen and Chiu's Fault Tolerant Routing Algorithm for Mesh Networks," J. Information Science and Eng., vol. 23, pp. 1649-1662, May 2007.

[18] J. Hu and R. Marculescu, "Energy-Aware Mapping for Tile-Based NoC Architectures under Performance Constraints," Proc. ASP Design Automation Conf., pp. 233-239, Jan. 2003.

[19] S.R. Sridhara and N.R. Shanbhag, "Coding for System-on-Chip Networks: A Unified Framework," IEEE Trans. Very Large Scale Integration (VLSI) Systems, vol. 13, pp. 655-667, June 2005.

[20] C. Duan, A. Tirumala, and S.P. Khatri, "Analysis and Avoidance of Cross-Talk in On-Chip Buses," Proc. IEEE Symp. HighPerformance Interconnects, pp. 133-138, Aug. 2001.

[21] S. Murali and G. De Micheli, "Bandwidth-Constrained Mapping of Cores onto NoC Architectures," Proc. Design, Automation and Test in Europe Conf. and Exhibition, vol. 2, pp. 896-901, Feb. 2004.

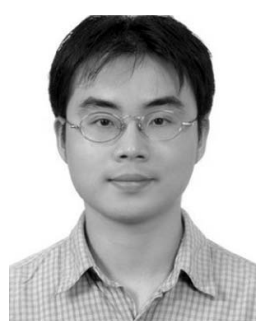

Shu-Yen Lin received the BS degree in electronic engineering from $\mathrm{Fu}$ Jen Catholic University, Taiwan, in 2002, and the MS degree in electronic engineering from the National Taiwan University of Science and Technology, Taiwan, in 2004. He is currently working toward the $\mathrm{PhD}$ degree at the Graduate Institute of Electronics Engineering, National Taiwan University, Taipei. His research fields include the architecture and algorithm design for on-chip networks. His research interests are in the areas of on-chip networks, lossless data compression, and fault-tolerant designs.

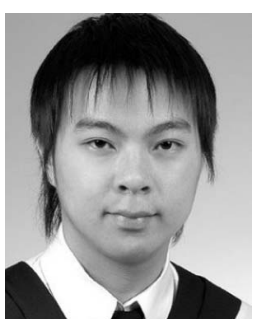

Chun-Hsiang Huang received the BS and MS degrees in electrical engineering from National Taiwan University, Taipei, in 2005 and 2007, respectively. $\mathrm{He}$ is currently an engineer at Realtek Semiconductor Corp., Hsinchu, Taiwan, where he is working on communication IC designs.

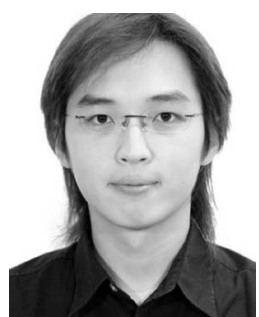

Chih-Hao Chao received the BS and MS degrees in electrical engineering from National Taiwan University, Taipei, in 2004 and 2006, respectively. He is currently working toward the PhD degree at the Graduate Institute of Electronics Engineering, National Taiwan University, Taipei. His research fields include algorithm and architecture design for location estimation and ESL design methodology. 


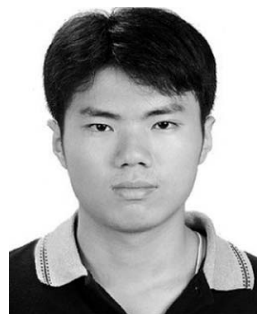

Keng-Hsien Huang received the BS degree in electrical engineering from National Taiwan University, Taipei, in 2007, respectively. $\mathrm{He}$ is currently pursuing the MS degree from the Graduate Institute of Electronics Engineering, National Taiwan University, Taipei.

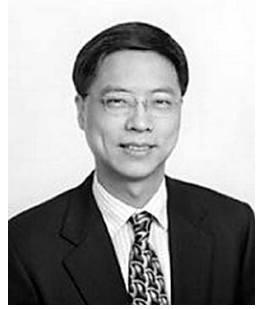

An-Yeu (Andy) Wu received the BS degree in electrical engineering from National Taiwan University in 1987 and the MS and PhD degrees in electrical engineering from the University of Maryland, College Park, in 1992 and 1995, respectively. From August 1995 to July 1996, he was a member of the technical staff at AT\&T Bell Laboratories, Murray Hill, New Jersey, working on high-speed transmission IC designs. From 1996 to July 2000, he was with the Electrical Engineering Department at National Central University, Taiwan. In August 2000, he joined the faculty of the Department of Electrical Engineering and the Graduate Institute of Electronics Engineering at National Taiwan University, where he is currently a professor. His research interests include low-power/high-performance VLSI architectures for DSP and communication applications, adaptive/ multirate signal processing, reconfigurable broadband access systems and architectures, and SoC platform for software/hardware codesign. He served as an associate editor for the EURASIP Journal of Applied Signal Processing from 2001 to 2004 and acted as the leading guest editor for a special issue on "Signal Processing for Broadband Access Systems: Techniques and Implementations" of the same journal (published in December 2003). He also served as an associate editor for the IEEE Transactions on Very Large Scale Integration (VLSI) Systems from 2003 to 2005. He is now an associate editor of the IEEE Transactions on Circuits and Systems I: Regular Papers. He has served on the technical program committees of many major IEEE International Conferences such as ICIP, SiPS, AP-ASIC, ISCAS, ISPACS, ICME, SOC, and A-SSCC. He received the A-class Research Award from National Science Council four times from 1997 to 2000 . He received the Macronix International Corp. Young Chair Professor Award in 2003. In 2004, he received the Distinguished Young Engineer Award from the Chinese Institute of Electrical Engineering, Taiwan. In 2005, he received two research awards, the Dr. Wu Ta-you Award (young scholar award) and the President Fu Si-nien Award, from the National Science Council and National Taiwan University, respectively, for his research work in VLSI system designs. Since August 2007, he has been on leave, serving as the deputy general director of the SoC Technology Center (STC), Industrial Technology Research Institute (ITRI), Hsinchu, Taiwan. $\mathrm{He}$ is a member of the IEEE.

$\triangleright$ For more information on this or any other computing topic, please visit our Digital Library at www.computer.org/publications/dlib. 